\title{
Author Correction: Time-restricted feeding restores muscle function in Drosophila models of obesity and circadian-rhythm disruption
}

\author{
Jesús E. Villanueva, Christopher Livelo, Adriana S. Trujillo, Sahaana Chandran, Brendon Woodworth (D), \\ Leo Andrade, Hiep D. Le, Uri Manor (1), Satchidananda Panda \& Girish C. Melkani (1)
}

Correction to: Nature Communications https://doi.org/10.1038/s41467-019-10563-9, published online 20 June 2019

The original version of this Article contained errors in confocal images in Fig. 3, Fig. 4, and Supplementary Fig. 3. During the first and second resubmissions of this article, the images in question were accidentally improperly named when imported to Power Point for figure preparation which led to several duplications.

The following figure panels were inadvertently and erroneously duplicated: Figure 1h panel "WT-HFD" was duplicated in Fig. 3c as panel "Fln $>$ Sk2KD, 3 W"; Fig. 8h panel "WT TRF-LL" was duplicated in Fig. 3c as panel "Fln/+, 3 W"; Fig. 8h panel "ALF-LL WT" was duplicated in Fig. 4c as panel "Bmm/+, 3 W"; Fig. 6e panel "TRF WT-RD” was duplicated in Fig. 4c as panel "CS control, 3 W".

Panel "Fln/+, 3 W" in Fig. 3c and panel "WT + bromoenol lactone" in Supplementary Fig. 3a were identical and incorrect.

Furthermore, the Y-axis label in Fig. 3b was absent and should have stated "Relative NLaz Expression". This has now been added. 
The correct version of Fig. 3 is:
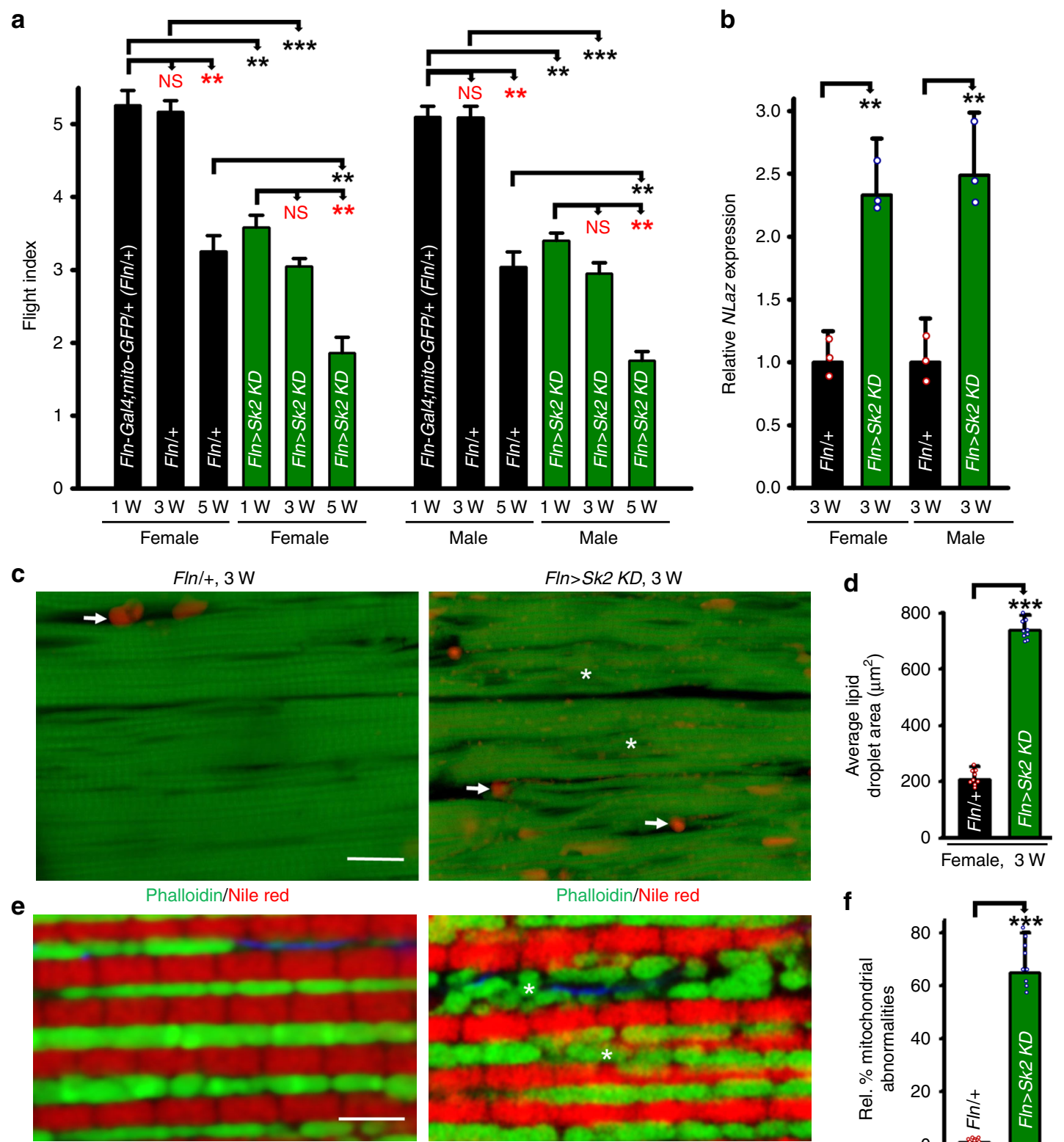

Phalloidin/Nile red

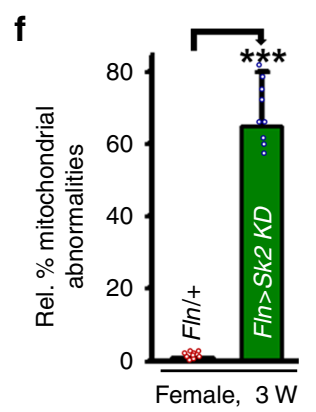

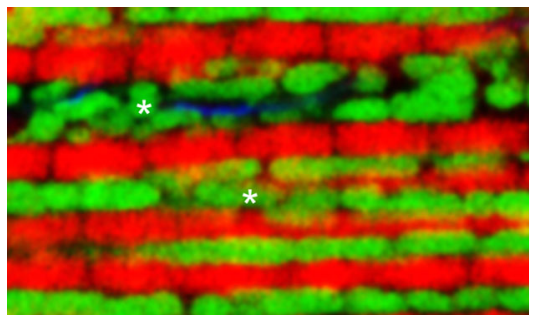

Phalloidin/mito-GFP/DAPI 
which replaces the previous incorrect version:
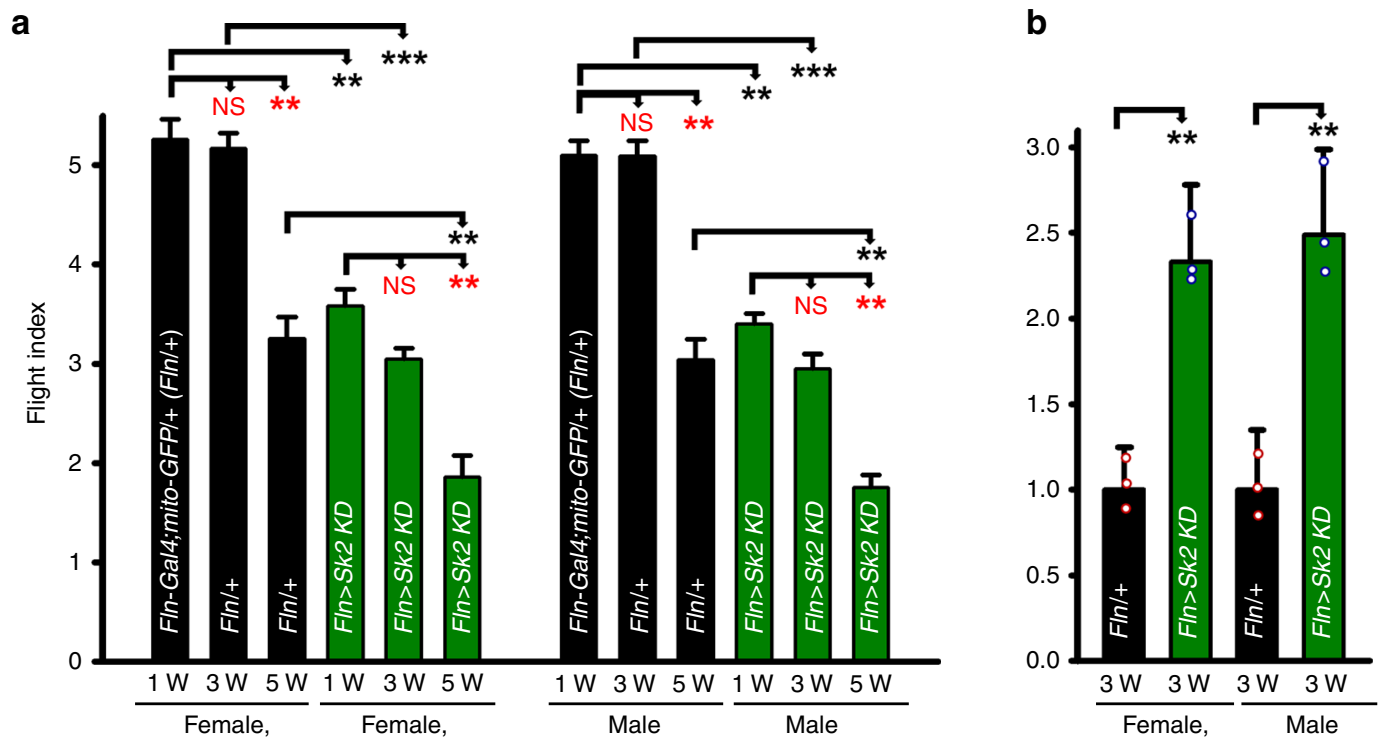

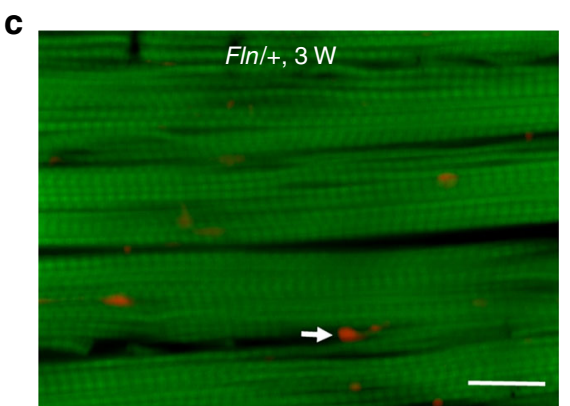

Phalloidin/Nile red

e

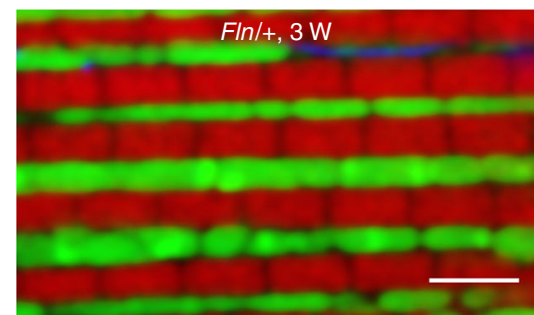

Phalloidin/mito-GFP/DAPI

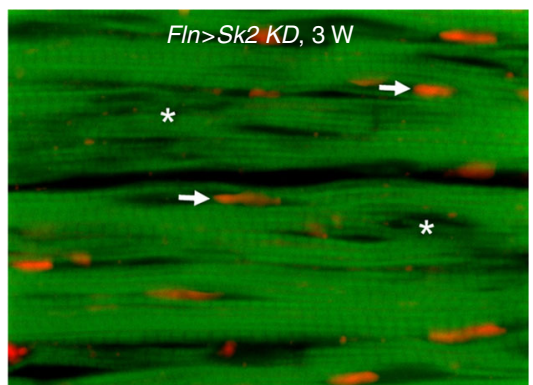

Phalloidin/Nile red

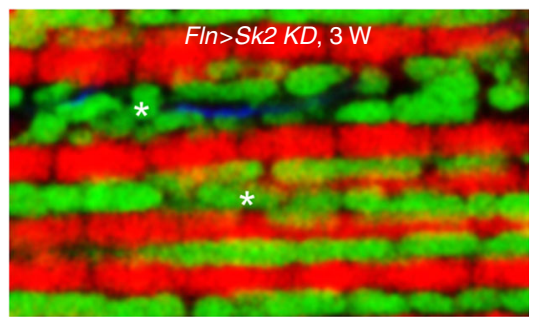

Phalloidin/mito-GFP/DAPI

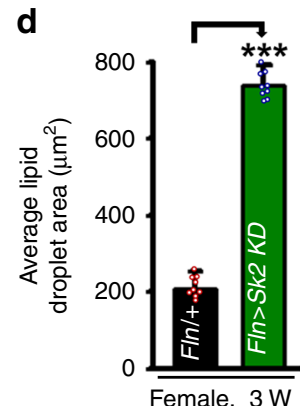

f

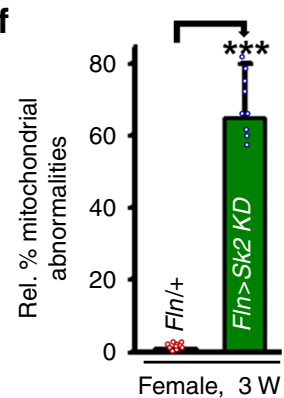


The correct version of Fig. 4 is:

a

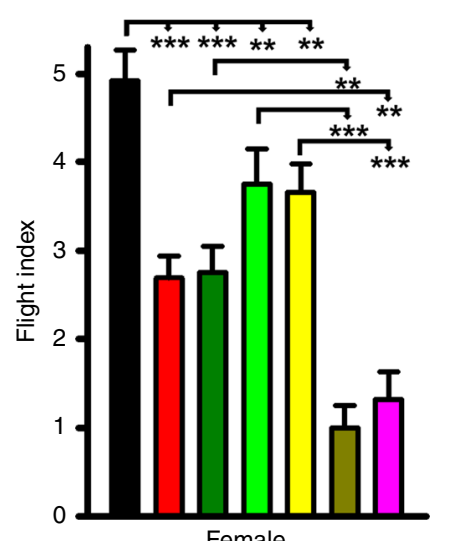

b

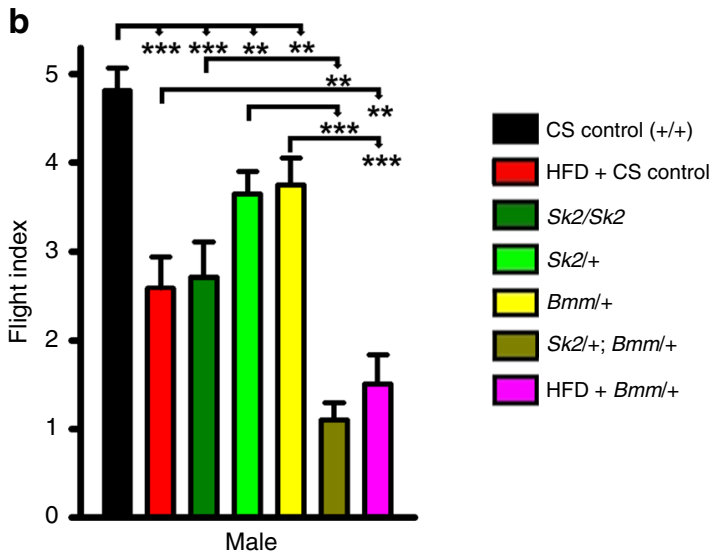

c

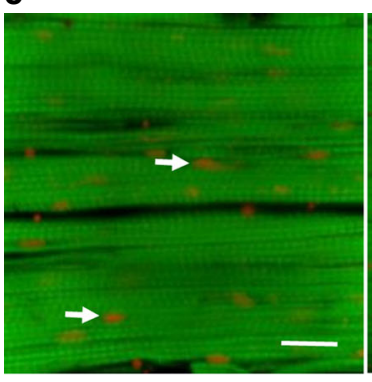

CS control (+/+), $3 \mathrm{~W}$

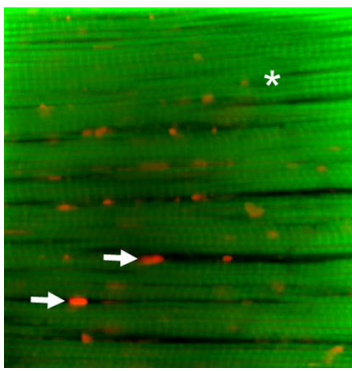

HFD + CS control, $3 \mathrm{~W}$

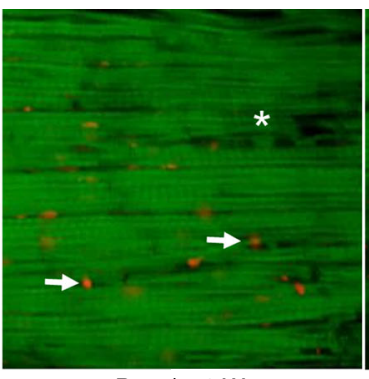

$B m m /+, 3 \mathrm{~W}$

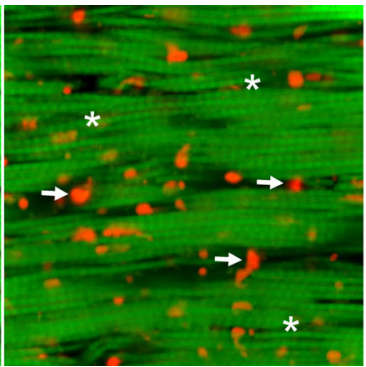

$\mathrm{HFD}+\mathrm{Bmm} /+, 3 \mathrm{~W}$

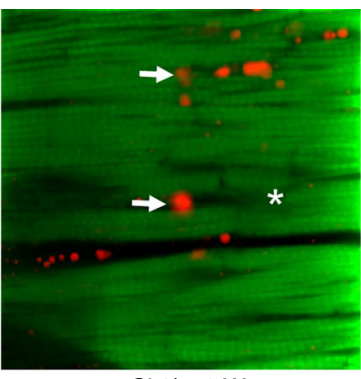

Sk2/+, $3 \mathrm{~W}$

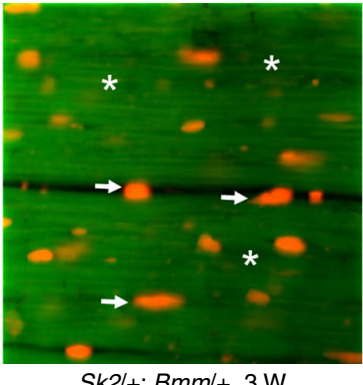

Sk2/+; Bmm/+, $3 \mathrm{~W}$

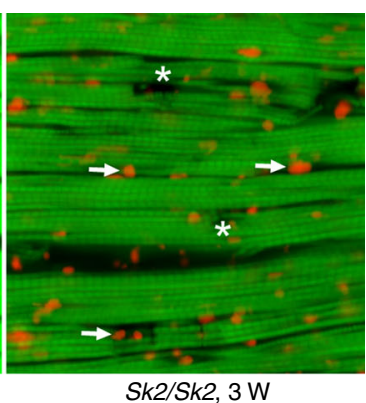

d 1200$]$ 
which replaces the previous incorrect version:

a

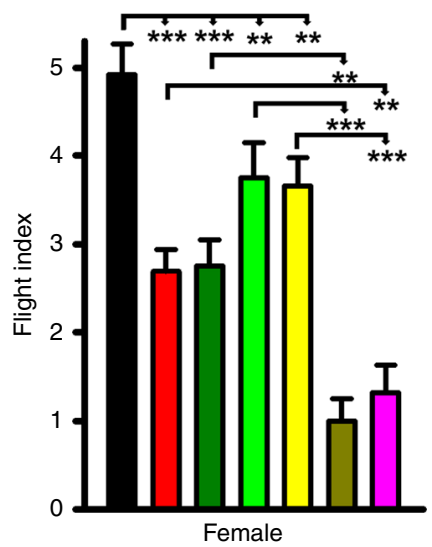

b

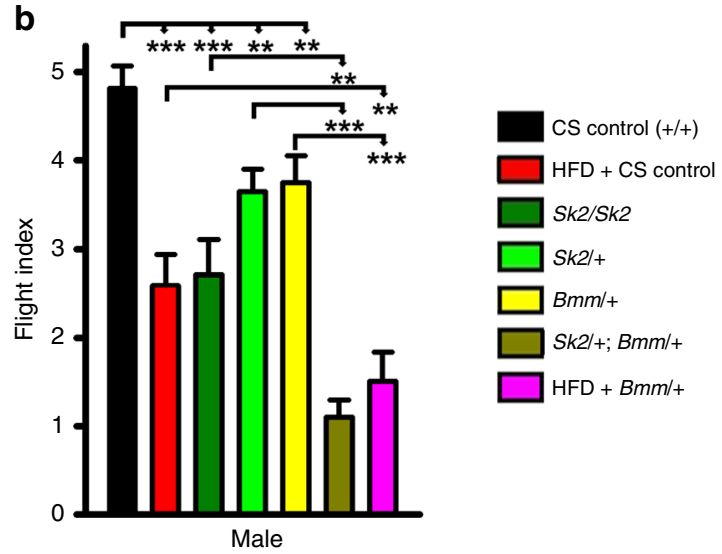

C

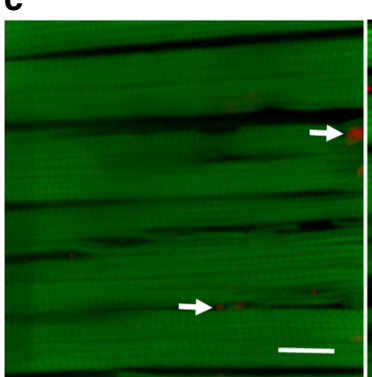

CS control $(+/+), 3 \mathrm{~W}$

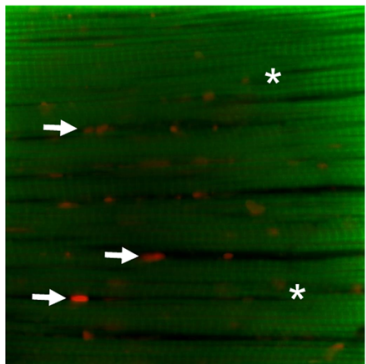

HFD + CS control, $3 \mathrm{~W}$

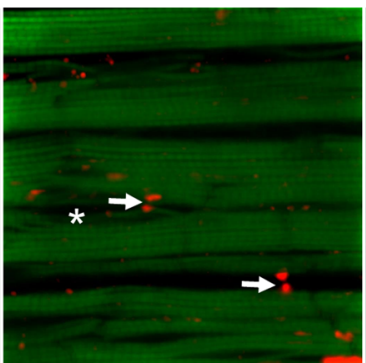

$\mathrm{Bmm} /+, 3 \mathrm{~W}$

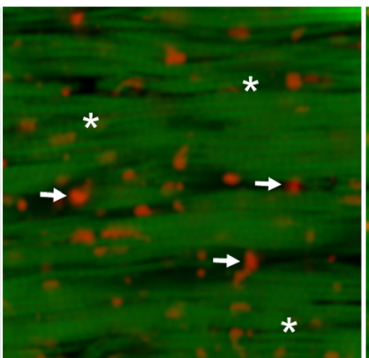

$\mathrm{HFD}+\mathrm{Bmm} /+, 3 \mathrm{~W}$

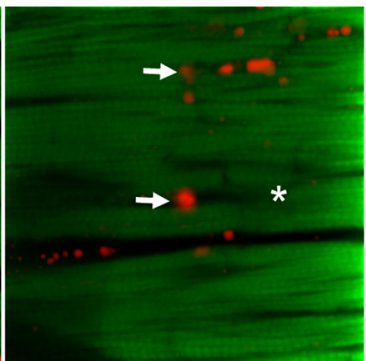

$S k 2 /+, 3 \mathrm{~W}$

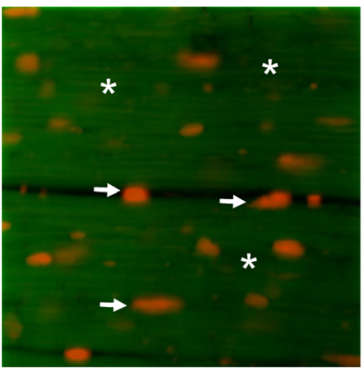

Sk2/+; Bmm/+, $3 \mathrm{~W}$

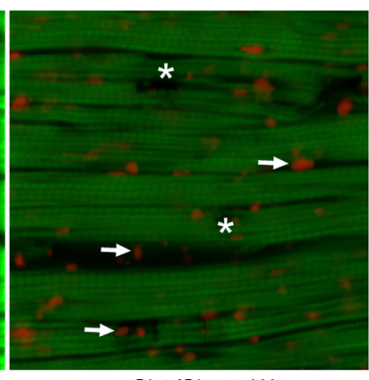

Sk2/Sk2, $3 \mathrm{~W}$

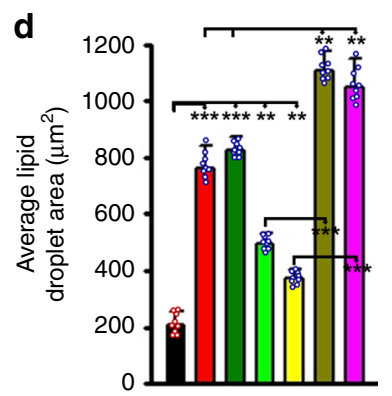

This has now been corrected in both the PDF and HTML versions of the Article.

In addition, the following duplicated panels have the same genotypes but have been replaced by other representative images for clarity: Fig. 6e and Supplementary Fig. 3a panel "WT-HFD ALF"; the image was replaced in Supplementary Fig. 3a. Figure 1h and Supplementary Fig. 3a panel "Sk2"; the image was replaced in Supplementary Fig. 3a.

The errors have now been fixed and the Supplementary Information PDF is available to download from the HTML version of the Article.

All original data for the images presented in these figures can be accessed using the Figshare link https:/figshare.com/s/ 682fflae4cde2f2729a4. Additional images from independent replicates can also be accessed using the Figshare link.

Published online: 15 May 2020

(c) Open Access This article is licensed under a Creative Commons Attribution 4.0 International License, which permits use, sharing, adaptation, distribution and reproduction in any medium or format, as long as you give appropriate credit to the original author(s) and the source, provide a link to the Creative Commons license, and indicate if changes were made. The images or other third party material in this article are included in the article's Creative Commons license, unless indicated otherwise in a credit line to the material. If material is not included in the article's Creative Commons license and your intended use is not permitted by statutory regulation or exceeds the permitted use, you will need to obtain permission directly from the copyright holder. To view a copy of this license, visit http://creativecommons.org/licenses/by/4.0/. 